\title{
Instrumento para avaliação da qualidade de prontuários de admissão em unidades de urgência e emergência
}

\author{
Instrument for assessing the quality of the admission records in urgent and emergency \\ units
}
Instrumento para evaluar la calidad de los registros de admisión en unidades de urgencia y emergencia

Stella Costa Todt ${ }^{1}$, Isabella Paiva Palmeira ${ }^{1}$, Lara Rebeca Correia Franca Dantas ${ }^{1}$, Carlos Alberto Miranda Lyra1 ${ }^{1}$, Maria Bernadete Galrão de Almeida Figueiredo ${ }^{1}$, Carla Viviane Freitas de Jesus, Sonia Oliveira Lima ${ }^{1 *}$.

\section{RESUMO}

Objetivo: Criar um instrumento que permita a classificação da qualidade dos prontuários de admissão de pacientes em unidades de urgência e emergência, verificando os itens mais omitidos e os mais avaliados no preenchimento dos prontuários. Métodos: Estudo metodológico, o qual analisou qualitativamente material impresso de 1195 prontuários eletrônicos coletados em uma instituição de urgência e emergência privada no Nordeste brasileiro. Utilizada amostra por conveniência de prontuários de pacientes com 18 anos ou mais e excluídos aqueles que não foram submetidos a avaliação médica. Os prontuários coletados foram avaliados pelo instrumento proposto, segundo os itens: identificação, caracterização de queixa principal, alergias, medicações em uso, antecedentes pessoais patológicos e gravidez, última refeição, motivo e hora da admissão, estado geral e exame físico. Resultados: Na classificação final proposta pelo instrumento, obtevese 88 prontuários completos, 773 básicos e 334 insuficientes. Ao analisar os dados coletados nesse estudo, ficou evidente uma falha na construção dos prontuários, principalmente nos itens estado geral, última refeição, medicamentos em uso, alergias e antecedentes pessoais. Conclusão: Portanto, torna-se relevante a utilização de um instrumento específico para a avaliação e classificação dos prontuários, otimizando o atendimento, o diagnóstico e o prognóstico dos pacientes, e protegendo todos os envolvidos no âmbito judicial.

Palavras-chave: Análise qualitativa, Medicina de emergência, Registros médicos.

\section{ABSTRACT}

Objective: To create an instrument that allows the classification of the quality of patient admission records in urgency and emergency units, verifying the most omitted and most evaluated items when filling out medical records. Methods: Methodological study, which qualitatively analyzed printed material from 1195 electronic medical records collected in a private urgent and emergency institution in Northeastern Brazil. A sample was used for the convenience of medical records of patients aged 18 years or older and those who did not undergo medical evaluation were excluded. The collected medical records were evaluated by the proposed instrument, according to the items: identification, characterization of the main complaint, allergies, medications in use, personal pathological history and pregnancy, last meal, reason and time of admission, general condition and physical examination. Results: In the final classification proposed by the instrument, 88 complete medical records, 773 basic and 334 insufficient ones were obtained. When analyzing the data collected in this study, a flaw in the construction of medical records was evident, mainly in the items general condition, last meal, medications in use, allergies, and personal history. Conclusion: Therefore, it becomes relevant to use a specific instrument for the evaluation and classification of medical records, optimizing patient care, diagnosis, and prognosis, and protecting all those involved in the judicial sphere.

Keywords: Qualitative analysis, Emergency medicine, Medical records.

1Universidade Tiradentes (UNIT), Aracaju - SE. *E-mail: sonialima.cirurgia@gmail.com 


\section{RESUMEN}

Objetivo: Crear un instrumento que permita clasificar la calidad de los registros de admisión de pacientes en las unidades de urgencia y emergencia, verificando los elementos más omitidos y evaluados al completar los registros médicos. Métodos: Estudio metodológico, que analizó cualitativamente material impreso de 1195 registros médicos electrónicos recopilados en una institución privada de urgencias y emergencias en el noreste de Brasil. Se utilizó una muestra para la conveniencia de los registros médicos de pacientes de 18 años o mayores y aquellos que no se sometieron a evaluación médica fueron excluidos. Los registros médicos recopilados fueron evaluados por el instrumento propuesto, de acuerdo con los ítems: identificación, caracterización de la queja principal, alergias, medicamentos en uso, antecedentes patológicos personales y embarazo, última comida, razón y tiempo de admisión, estado general y examen físico. Resultados: En la clasificación final propuesta por el instrumento, se obtuvieron 88 registros médicos completos, 773 básicos y 334 insuficientes. Al analizar los datos recopilados en este estudio, se evidenció una falla en la construcción de registros médicos, principalmente en los ítems estado general, última comida, medicamentos en uso, alergias e historial personal. Conclusión: Por lo tanto, se vuelve relevante utilizar un instrumento específico para la evaluación y clasificación de registros médicos, optimizando la atención, el diagnóstico y el pronóstico del paciente, y protegiendo a todos los involucrados en la esfera judicial.

Palabras clave: Análisis cualitativo, Medicina de emergência, Registros médicos.

\section{INTRODUÇÃO}

Prontuário, do latim promptuarium, significa "lugar onde são guardadas coisas de que se pode precisar a qualquer momento" ou "manual de informações úteis" ou ainda "ficha que contém os dados pertinentes de uma pessoa" (HOUAISS A, et al., 2009). O Conselho Federal de Medicina, caracteriza o prontuário como documento científico, legal e sigiloso, composto por informações referentes ao paciente e a assistência a ele prestada, o que permite a continuidade da avaliação, já que facilita a comunicação dentro da equipe multiprofissional (REIS AOA, et al., 2009).

O registro de informações dos enfermos remota a idade antiga, porém, foi com Hipócrates de Cós, no século V a.C. com a institucionalização da Medicina Científica, que foi evidenciada a real importância e necessidade de se fazer o registro escritos das informações sobre a saúde do paciente (PINTO VB, 2006). Esse posicionamento também foi arduamente defendido por Florence Nightingale, a precursora da enfermagem moderna, a qual defendia a necessidade de uma transformação nas condições e formas como eram atribuídas a saúde no final do século XIX e pronunciava que esses registros eram de extrema importância para a continuidade do tratamento do paciente (MARTINS DF e BENITO LAO, 2016).

O prontuário deve ser fonte de informações onde, como e quando o usuário julgar útil, tais informações possuem um amplo espectro de alcance: a mais evidente, na tomada de decisão médica; e as mais subentendidas, para fins de pesquisa e epidemiologia. Para o setor administrativo emitir relatórios de gerência, faturamento e proporcionar resguardo legal. A responsabilidade do preenchimento correto do prontuário não recai somente no profissional de saúde. Os gestores das unidades de urgência e emergência (UE) também devem garantir e fiscalizar a elaboração desse importante documento. Para isso, é fundamental que existam ferramentas capazes de uma avaliação qualitativa dos prontuários e instauração de medidas e protocolos para melhorar seu preenchimento (PATRÍCIO CM, et al., 2011; GARRITANO CRO, et al., 2020).

Existe uma necessidade local e global de pesquisas e dados epidemiológicos sobre a qualidade dos prontuários médicos. Os métodos para a avaliação qualitativa dessas histórias clínicas são precários, todavia, a literatura descreve padrões bem definidos de assistência às urgências e emergências. A compreensão dos mecanismos da enfermidade é fundamental para que o atendimento médico seja completo, em casos específicos que o paciente seja incapaz de comunicar-se com o médico, um familiar pode fornecer as informações necessária (MARX J, et al., 2013; VINCENT JL, 2016; RODRIGUES TO, et al., 2019).

Dentre as estratégias de atendimento de UE, uma das mais utilizadas hoje é a estratégia "AMPLA" que consiste em: alergias; medicamentos em uso; passado médico e gravidez; líquidos e alimentos ingeridos na última refeição; ambientes e eventos que levaram ao trauma. Esta guiará uma história clínica precisa (MARX J, et al., 2013; ASANZA DM, et al., 2018). 
A realização da triagem, com o preenchimento do prontuário, representa etapa fundamental no ambiente de urgência e emergência, onde os pacientes serão classificados, para posterior atendimento, de acordo com a gravidade da injúria ou enfermidade, disponibilidade de recursos para o tratamento e chance de recuperação (ATLS, 2008). Os registros desta etapa são primordiais para um bom curso da assistência, visto que podem interferir na complexidade da conduta, na agilidade do tratamento e local para o qual o paciente será encaminhado, ao ponto de resultar em desclassificação do mesmo do atendimento de urgência. Assim sendo, o profissional responsável por esta fase do atendimento tem o dever de preencher o prontuário de modo meticuloso, corroborando em uma triagem justa que reflita a realidade do indivíduo (BECKER JB, et al., 2015; AMTHAUER C e CUNHA MLC, 2016).

O presente estudo teve como objetivo criar um instrumento que permita a classificação da qualidade dos prontuários de admissão de pacientes em unidades de urgência e emergência, verificando os itens mais omitidos e os mais avaliados no preenchimento dos prontuários.

\section{MÉTODOS}

A abordagem foi constituída por um estudo metodológico com desenvolvimento de instrumento para avaliação qualitativa de prontuários de admissão de pacientes em setor de UE.

\section{População e Amostra}

Amostra composta por conveniência de prontuários de pacientes com idade de 18 anos ou mais admitidos no setor de UE de um hospital privado do nordeste brasileiro entre Janeiro de 2017 e Março de 2017. Foram excluídos os prontuários de pacientes que não foram submetidos a avaliação médica.

\section{Local da Pesquisa}

Unidade de urgência e emergência, com capacidade de 18 leitos e 19 poltronas, de um hospital de rede privada em Aracaju-Sergipe, que recebeu a partir do ano de 2016 a acreditação Internacional Canadense Qmentun Diamante e nível três pela Organização Nacional de Acreditação.

\section{Sistemática e Instrumentos de Coleta de Dados}

A coleta dos dados foi realizada pelos autores, com treinamento prévio, em material impresso de prontuário eletrônico. Os prontuários foram graduados mediante critérios validados por 5 médicos com experiência de no mínimo 10 anos no atendimento de UE, de acordo com a sua qualidade em: insuficiente, básico e estendido; e ausente ou presente. Os itens avaliados foram: identificação, caracterização de queixa principal, alergias, medicações em uso, antecedentes pessoais patológicos e gravidez, última refeição, motivo de admissão, hora da admissão, estado geral e exame físico (Tabela 1).

$\underline{\text { Tabela } 1 \text { - Grau de qualidade dos critérios avaliados }}$

\begin{tabular}{cccc}
\hline Critérios & $\mathbf{0}$ & $\mathbf{1}$ & $\mathbf{2}$ \\
\hline Identificação & Insuficiente & Básico & Estendido \\
Caracterização da queixa principal & Insuficiente & Básico & Estendido \\
Alergias & Ausente & Presente & \\
Medicações & Ausente & Presente & \\
Antecedentes Pessoais (Patológicos/ Gravidez) & Ausente & Presente & \\
Última refeição & Ausente & Presente & \\
Motivo de admissão & Ausente & Presente & \\
Hora de Admissão & Ausente & Presente & \\
Estado geral & Ausente & Presente & \\
Exame físico & Insuficiente & Básico & Estendido \\
\hline
\end{tabular}

Fonte: Todt SC, et al., 2020.

A determinação entre básico e estendido dos itens, abordados no instrumento, foi feita respeitando os seguintes aspectos: para a identificação, os prontuários que apresentaram nome e idade foram classificados como básicos; já os que além dos dados contemplados no básico, apresentaram também pelo menos três dados extras (profissão, religião, naturalidade, procedência, residência, sexo/gênero, etnia, estado civil, nome da mãe, plano de saúde) foram classificados como estendidos. 
No item de caracterização da queixa principal: foram classificados como básicos os prontuários que continham registros das características do sintoma, circunstâncias de início e situação atual; os que além de preencherem os critérios para ser básico, também apresentaram pelo menos dois de três dados extras (fatores de melhora e piora, relação com outras queixas e evolução) foram classificados qualitativamente como estendidos. Na etapa do exame físico, foram graduados em básico os que contemplavam o exame do órgão e/ou sistema afetado; e como estendido, os que além do básico, realizavam também o exame geral.

$\mathrm{Na}$ Tabela 2, pesos foram aferidos a cada critério, visto que os mesmos possuem graus de relevância distintos entre si. Na Tabela 3, é apresentada a classificação final de acordo com a pontuação bruta e, ponderada, obtém-se um dos resultados: insuficiente, [0-5]; básico, [6-10]; ou completo, [11-13].

Tabela 2 - Pesos dos critérios avaliados.

\begin{tabular}{cc}
\hline Critérios & Pesos \\
\hline Identificação & 2 \\
Caracterização da queixa principal & 1 \\
Alergias & 2 \\
Medicações & 2 \\
Antecedentes Pessoais (Patológicos/Gravidez) & 1 \\
Última refeição & 1 \\
Motivo de admissão & 2 \\
Hora de Admissão & 1 \\
Estado geral & 1 \\
\hline
\end{tabular}

Fonte: Todt SC, et al., 2020.

Tabela 3 - Classificação Final

\begin{tabular}{cccc}
\hline Pontuação bruta & Completo & Básico & Insuficiente \\
\hline & {$[11-13]$} & {$[6-10]$} & {$[0-5]$} \\
\hline Pontuação ponderada & Completo & Básico & Insuficiente \\
\hline & {$[11-13]$} & {$[6-10]$} & {$[0,5]$} \\
\hline
\end{tabular}

Fonte: Todt SC, et al., 2020.

\section{Análise de Dados}

Analisou-se os prontuários em médias simples, desvio padrão e erro padrão da média utilizando o programa Microsoft Excel versão 15.13.3.

\section{Aspectos éticos}

O trabalho foi realizado de acordo com as normas e diretrizes 510/2016 e aprovado pelo Comitê de Ética em Pesquisa da Universidade Tiradentes com parecer número 2.226.720.

\section{RESULTADOS}

Identificou-se 1195 prontuários do período de Janeiro a Março de 2017. Os itens identificação, motivo de admissão e hora de admissão tem padronização da instituição pesquisada, e foram pontuados com um ponto no instrumento proposto. Foram encontradas médias simples de $1,25 \pm 0,020$ para a caracterização da queixa dos pacientes, $0,45 \pm 0,014$ para alergias, $0,29 \pm 0,013$ para medicamentos em uso, $0,50 \pm 0,014$ para

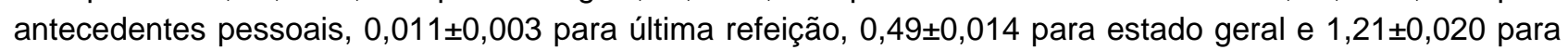
descrição do exame físico. Os resultados das médias da pontuação bruta podem ser vistos na Figura 1. 
Figura 1 - Médias e desvio padrão dos resultados obtidos na coleta de 1195 prontuários, de pacientes atendidos entre Janeiro de 2017 a Março de 2017, hospital particular em Aracaju-SE.

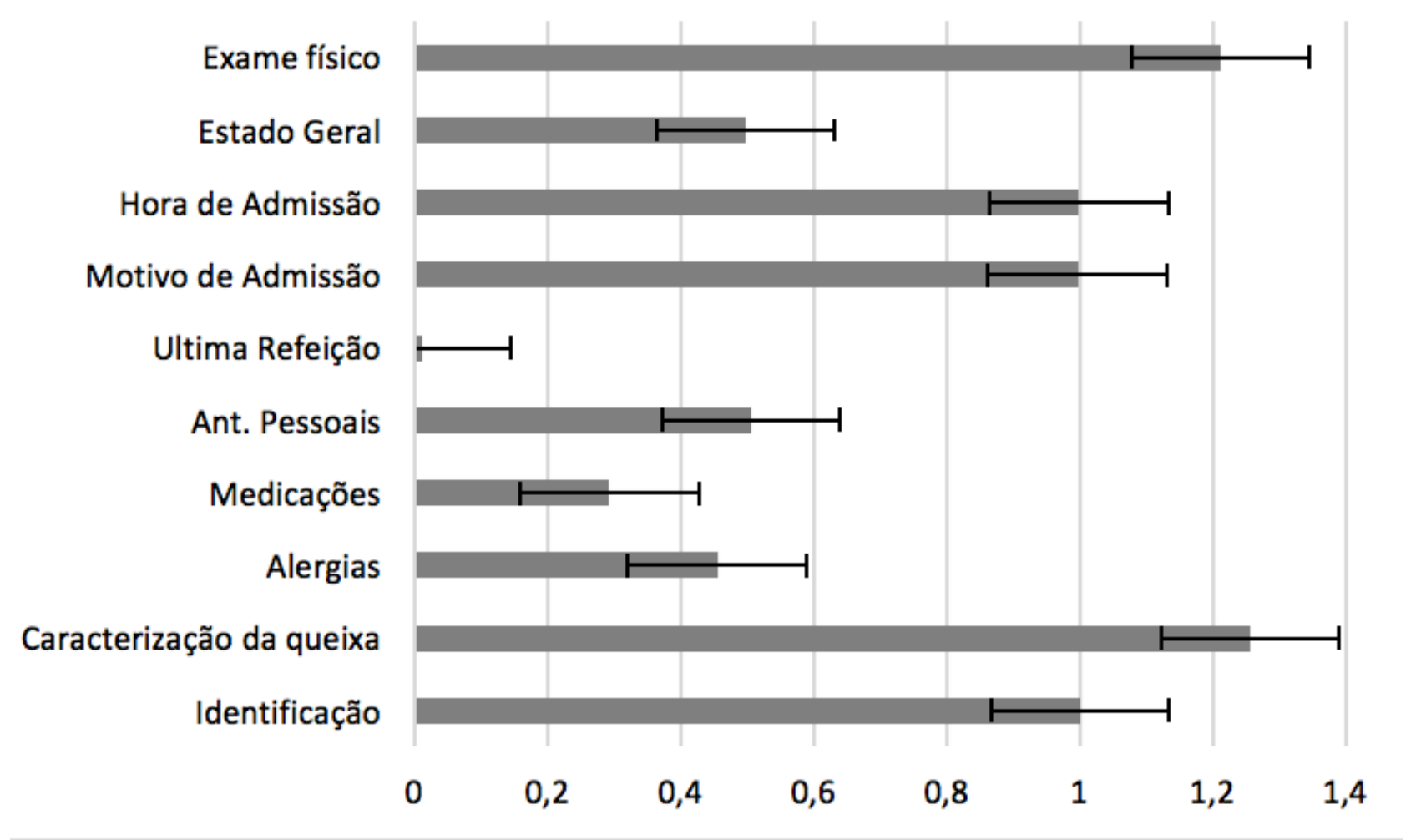

Fonte: Todt SC, et al., 2020.

Na classificação final dos prontuários, a média geral da pontuação bruta foi de 7,22 $\pm 0,065$ com desvio padrão de 2,25 classificando-os como básicos. Individualmente, 88 prontuários foram avaliados como completos, 773 como básicos e 334 como insuficientes (Figura 2).

Figura 2 - Distribuição em porcentagem da classificação final dos 1195 prontuários, de pacientes atendidos entre Janeiro de 2017 a Março de 2017, hospital particular em Aracaju-SE.

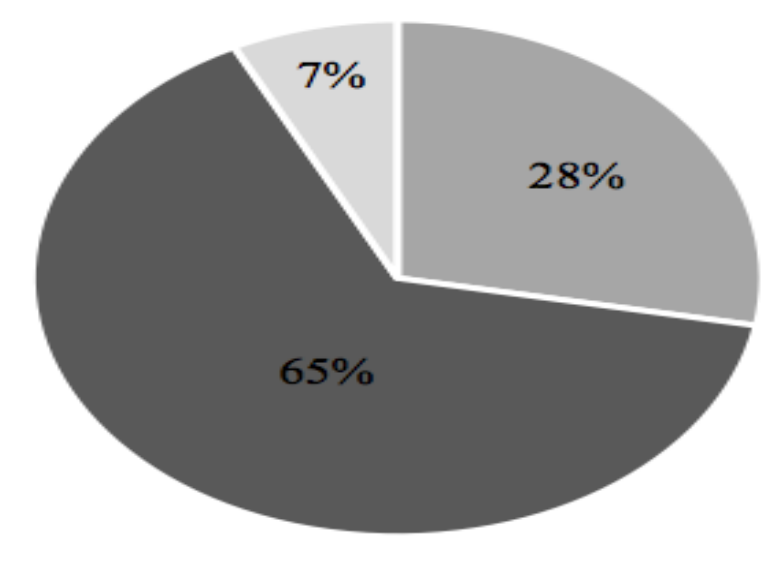

- Incompleto $\quad$ Basico $=$ Completo

Fonte: Todt SC, et al., 2020. 


\section{DISCUSSÃO}

Neste estudo o item identificação, obteve um ponto, classificado básico no instrumento proposto. O primeiro passo para a construção do atendimento e relação médico-paciente, em UE, é realizar a identificação do enfermo, a qual permite a interpretação de dados individuais e coletivos. A identificação precoce no ambiente de UE é de extrema importância, pois permite que os profissionais de saúde atuem com maior agilidade e precisão, prevenindo possíveis complicações (VINCENT JL, 2016; HAMMES AH, 2019). Portanto, a identificação classificada como básica não supre totalmente as necessidades do atendimento no ambiente hospitalar. Para a sua otimização é sugerido ampliar as informações de identificação contemplando profissão, religião, naturalidade, procedência, residência, sexo/gênero, etnia, estado civil, nome da mãe e plano de saúde.

A queixa principal (QP) é a transcrição do motivo da admissão no ambiente hospitalar. No presente estudo a caracterização da queixa atingiu uma média simples de $1,25 \pm 0,020$, a qual infere que a maioria dos prontuários avaliados apresentaram uma pontuação básica. Tal item é considerado uns dos mais importantes da coleta da história clínica, onde é detalhado todos os aspectos do motivo de admissão do paciente, desde o começo do sintoma, fatores de interferência, evolução e estado atual. Em ambientes de UE, a história clínica associada aos mecanismos de lesão, são críticos para a identificação do processo lesivo que está acometendo o paciente, permitindo assim, tomadas concretas de decisão (MARX J, et al., 2013; PORTO CC, 2014; AMORIM HPL, et al., 2016). Para o aperfeiçoamento da construção da QP, além dos itens básicos, devem ser implementados fatores de melhora e piora, relação com outras queixas e evolução.

No item alergias encontrou-se uma média simples de $0,45 \pm 0,014$, o que significa que menos da metade dos prontuários avaliados contemplaram esse componente. Identificar a existência de alergias, principalmente medicamentosas, é indispensável na coleta da história clínica. A importância desse item é o risco de complicações potencialmente fatais, como o choque anafilático (ENSINA LF, et al., 2009; MARTINS L, et al., 2019). É imprescindível a sua presença na formulação da história clínica, principalmente no ambiente de UE Assim, deve-se estimular métodos de padronização para a coleta dessa informação e diminuir as complicações causadas pela não investigação de alergias prévias.

Outro item que chama atenção por possíveis complicações é o de medicações em uso. Constatou-se uma média simples de $0,29 \pm 0,013$, a qual demonstra um importante déficit em sua coleta. A importância desse item também é comprovada pela necessidade de questioná-la na estratégia "AMPLA", indicando sua utilização indispensável no ambiente de UE. Além disso, as reações adversas a medicamentos podem estar relacionadas com os sintomas do paciente e demandam atenção dos profissionais de saúde (MARX J, et al., 2013; SWARTZ MH, 2015; SPANEVELLO S, et al., 2018; VAIDOTAS M, et al., 2019). Portanto, por interferirem no processo de interação com outras drogas que possam ser usadas e gerar sintomas no paciente, é necessário a investigação dos medicamentos em uso a dose, a via de administração e a frequência de utilização.

Obteve-se no item antecedentes pessoais uma média simples de $0,50 \pm 0,014$, que reflete uma falha na construção da história clínica. Este item avalia o estado de saúde, passado e presente, do paciente e reconhece fatores pessoais e familiares que influenciam no processo saúde-doença. Abrange questionamentos sobre doenças anteriores, lesões, hospitalização, cirurgias, alergias, imunização, história obstétrica e medicamento em uso. Outro questionamento importante é a possibilidade de gravidez, por interferir na fisiologia, muitas vezes sendo um fator para complicações em uma série de doenças. Além disso, certos cuidados devem ser tomados no tratamento de uma grávida, no intuito de não causar nenhum dano ao bebê (BICKLEY LS, 2014; SWARTZ MH, 2015; DESAI B e DESAI A, 2016). Portanto, os antecedentes pessoais devem ser sempre contemplados no ambiente de UE, visto as possíveis variáveis decorrentes da individualidade de cada ser.

No questionamento da última refeição foi encontrada uma média $0,011 \pm 0,003$, que reflete o fato de quase $100 \%$ das histórias clínicas não abordaram esse item. A importância da pesquisa da última refeição, em relação ao seu conteúdo, quantidade e principalmente tempo entre a ingesta e a ocorrência, está atrelada ao fato de determinados alimentos serem capazes de provocar consequências no organismo que possam vir a 
agravar o quadro clínico do paciente, onde diagnóstico e conduta imediatas são determinantes para sobrevivência. Ademais, nos casos em que a conduta adotada for cirúrgica, esse dado torna-se ainda mais relevante (ATLS, 2018). Percebeu-se um descaso na coleta desse item por muitos profissionais de saúde.

Os dados de motivo e hora de admissão possuem caráter primário e norteador não apenas para o médico, mas também para os demais profissionais que forem consultar o prontuário seja durante o período de internação do paciente ou, posteriormente, por razões de revisão do caso; viés jurídico; ou para fins de estudo e pesquisa (PORTO CC, 2014). Satisfatoriamente, devido a padronização da instituição, todos prontuários obtiveram a pontuação máxima no score, o que corrobora o fato da importância da existência de padronizações nos ambientes hospitalares.

O principal objetivo da anamnese é arrecadar todas as informações básicas pertinentes ao paciente, de forma concatenada e de fácil compreensão não somente para o médico que a fez, mas para toda a equipe de saúde que possa vir a consultá-la (SWARTZ MH, 2015; MICHELS L, 2018). As informações contidas na transcrição da história clínica devem abranger não somente perguntas feitas ao paciente, mas também a observação médica da expressão corporal e facial, do comportamento e postura do enfermo. Verificou-se nessa pesquisa que a maioria dos itens referentes à anamnese necessita de uma melhor padronização, no intuito de otimizar o atendimento e o fluxo dos pacientes. Uma anamnese mal estruturada a partir da omissão de dados, pode repercutir negativamente na evolução do paciente, a chance de eventos adversos é elevada, sendo a maioria deles previsíveis e passíveis de correções se estes forem considerados.

O item "estado geral" é obtido por meio da percepção subjetiva do médico diante da aparência do paciente, fundamentada em características como: nível de consciência e fala; sinais vitais; mobilidade; fácies; e comorbidades. Pode ser classificado em bom, regular ou mau estado geral e sua presença no prontuário é importante para estabelecer a evolução do paciente de forma sucinta (SWARTZ MH, 2015; MICHELS L, 2018). Foi encontrada uma média simples de $0,49 \pm 0,014$ para o item estado geral, que demonstra coleta insatisfatória desse item primordial, não somente para a determinação da conduta médica, mas também para sua evolução. Justifica-se a necessidade de uma padronização da construção de uma história clínica que contemple as necessidades requisitadas no ambiente de UE.

O item exame físico visa fornecer hipóteses diagnósticas e auxiliar na tomada de decisão. Como fundamento, possui quatros princípios: inspeção, ausculta, percussão e palpação; que são técnicas semiológicas para avaliação dos sistemas do organismo. No presente estudo esse item apresentou uma

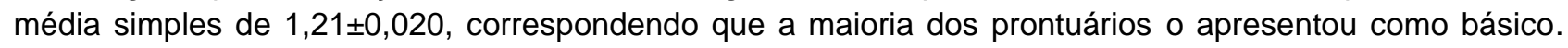
Todavia o cenário de UE exige uma abordagem ágil, direcionada e completa. No tocante à organização desse item no prontuário, há uma maior demanda por estabelecimento de padrões, tais como: ordem constante na disposição dos achados; títulos objetivos; destacar pontos importantes; e utilizar espaçamentos e parágrafos; padrões estes que acabam por facilitar e agilizar a compreensão do próprio médico ou de outros leitores (MOTA FRL, 2006; SWARTZ MH, 2015; BARRETO SP, et al., 2017). Portanto, na UE o exame físico básico não é ideal para avaliação do paciente podendo negligenciar outros achados para determinação do diagnóstico e conduta. Assim, um exame ideal, não deve explorar apenas a QP, mas, consistir em um exame físico geral.

Verificou-se, na avaliação geral proposta pelo instrumento, que a média simples da pontuação bruta e ponderada dos prontuários foram classificados como básicos. O que demonstrou, que mesmo possuindo carências, de modo geral as histórias clínicas avaliadas apresentaram um padrão de qualidade básica. Com essa avaliação qualitativa, visualizou-se as principais carências nos atendimentos de UE, de suas repercussões e significados. Individualmente, $7 \%$ dos prontuários foram avaliados como completos, $65 \%$ como básicos e $28 \%$ como insuficientes. Contudo, no ambiente de UE, o padrão deve ser prontuários classificados como completos, os quais nesse estudo, representaram a minoria das histórias clínicas avaliadas.

Os centros de UE são caracterizados por um nível elevado de estresse, alta demanda por agilidade e instabilidade de situações. Os aspectos citados constroem um cenário favorável para um preenchimento de prontuários mais suscetível à desatenção, falta de detalhamento e padronização. Assim sendo, as características inerentes aos setores de UE justificam a necessidade de critérios elaborados para avaliar 
qualitativamente tal documento (GABA DM e HOWARD SK, 2002). Prontuários classificados como básicos ou incompletos podem causar, direta ou indiretamente, uma série de danos as três principais entidades, 0 paciente, o médico e a instituição. A primeira, é a que está mais exposta e sensível a repercussões negativas em decorrência de condutas médicas baseadas em prontuários mal elaborados. Para o médico, o prontuário é um documento de valor jurídico, e um relato detalhado de como foi a sua conduta perante o enfermo, podendo ser utilizado em situações adversas ou ao seu favor (GARRITANO CRO, et al., 2020). A Instituição, como a UE, tem em seu arquivo o subsídio para monitorar os atendimentos, manter determinado padrão de qualidade, fazer uso em âmbito jurídico e fornecer dados para pesquisas.

Esta pesquisa alerta sobre a relevância da utilização de uma ferramenta capaz de avaliar e classificar os prontuários, dando ênfase para a busca de um padrão de qualidade elevado. A utilização do instrumento proposto deve ser estimulada no âmbito científico, visto que há escassez de artigos pertinentes a esse tema, com o intuito de possibilitar a criação de métodos de padronização no atendimento das UE e evidenciar os prejuízos que ocorrem pelos itens omitidos nesse documento. Destaca-se que os prontuários também servem como matéria-prima de pesquisas científicas, assim como permitem a análise da instituição sobre os serviços prestados. Deve-se alcançar um nível de excelência elevada na construção dos prontuários pelos médicos, com o intuito de otimizar o atendimento e reduzir os eventos adversos.

\section{CONCLUSÃO}

Foi possível a criação de um instrumento de avaliação da qualidade dos prontuários de admissão em serviços de urgência e emergência. A utilização desse instrumento permitiu verificar que houve negligência principalmente nos itens estado geral, última refeição, medicamentos em uso, alergias e antecedentes pessoais. A falha em colher esses dados, no prontuário, aumenta a possibilidade de surgirem complicações no decorrer do tratamento e na estadia do paciente no ambiente hospitalar. Portanto, torna-se relevante a utilização de um instrumento específico para a avaliação e classificação dos prontuários, otimizando o atendimento, o diagnóstico e o prognóstico dos pacientes, protegendo o paciente, além do médico e da instituição hospitalar, no âmbito judicial.

\section{REFERÊNCIAS}

1. AMERICAN COLLEGE OF SURGEONS (ATLS). Advanced Trauma Life Support: Student Course Manual. 10. ed. Chicago: American College of Surgeons; 2018; 391p.

2. AMORIM HPL, et al. A importância do preenchimento adequado dos prontuários para evitar processos em Odontologia. Arquivos em Odontologia, 2016; 52(1): 32-37.

3. AMTHAUER C, CUNHA MLC. Sistema de Triagem de Manchester: principais fluxogramas, discriminadores e desfechos dos atendimentos de uma emergência pediátrica. Revista Latino-Americana de Enfermagem, 2016; 24: e2779.

4. ASANZA DM, et al. Propuesta de un modelo de historia clínica ambulatoria en la urgencia estomatológica. Revista de Ciencias Médicas de Pinar del Río, 2018; 22(2): 94-102.

5. BARRETO SP, et al. Cateterismo cardíaco: um relato de experiência. Revista Rede de Cuidados em Saúde, 2017; 11(1): 1-5.

6. BECKER JB, et al. Triagem no Serviço de Emergência: associação entre as suas categorias e os desfechos do paciente. Revista escola de enfermagem da USP, São Paulo, 2015; 49(5): 783-789.

7. BICKLEY LS. BATES -Propedêutica médica. Guanabara Koogan, 2015; 758p.

8. DESAI B, DESAI A. Primary Care for Emergency Physicians. Springer, 2016; 324p.

9. ENSINA LF, et al. Reações de hipersensibilidade a medicamentos. Revista brasileira de alergia e imunopatologia, 2009; 32(2): 42-7.

10. GABA DM, HOWARD SK. Fatigue among clinicians and the safety of patients. New England Journal of Medicine, 2002; 347(16): 1249-1255.

11. GARRITANO CRO, et al. Avaliação do Prontuário Médico de um Hospital Universitário. Revista Brasileira de Educação Médica, 2020; 44(1): e:009.

12. HAMMES AH. Avaliação do preenchimento de prontuários médicos em um hospital geral. Dissertações (Bacharel em Medicina) Universidade Federal da Fronteira Sul, Passo Fundo, 2019; 42p.

13. HOUAISS A, et al. Dicionário Houaiss da língua portuguesa. Rio de Janeiro: Objetiva; 2009; 2048p.

14. MARTINS DF, BENITO LAO. Florence Nightingale e as suas contribuições para o controle das infecções hospitalares. Universitas: Ciências da Saúde, Brasília, 2016; 14(2): 153-166.

15. MARTINS L, et al. Prontuário Eletrônico do Paciente: Adoção de novas tecnologias de acesso. Journal of Health Informatics, 2019; 11(3): 67-73. 
16. MARX J, et al. Rosen's emergency medicine-concepts and clinical practice e-book. Elsevier Health Sciences, 2013; 2688p.

17. MICHELS L. Abdome agudo-importância da avaliação em pronto atendimento. Revista UNIPLAC, 2018; 6(1).

18. MOTA FRL. Prontuário eletrônico do paciente e o processo de competência informacional. Encontros Bibli: revista eletrônica de biblioteconomia e ciência da informação, 2006; 22: 53-70.

19. PATRíCIO CM, et al. O prontuário eletrônico do paciente no sistema de saúde brasileiro: uma realidade para os médicos. Scientia Medica, 2011; 21(3); 121-31.

20. PINTO VB. Prontuário eletrônico do paciente: documento técnico de informação e comunicação do domínio da saúde. Encontros Bibli: revista eletrônica de biblioteconomia e ciência da informação, 2006; 17(21): 34-48.

21. PORTO CC. Semiologia médica. In: Semiologia médica. Guanabara Koogan, 2014; 1360p.

22. REIS AOA, et al. Prontuários, para que servem? representação dos coordenadores de equipe dos capsi a respeito do valor e da utilidade dos prontuários. Journal of Human Growth and Development, 2009; 19(3): 383-392.

23. RODRIGUES TO, et al. Auditoria em prontuários como instrumento de qualidade da assistência: uma revisão da literatura. Journal of Specialist, 2019; 1(3).

24. SPANEVELLO S, et al. Interações medicamentosas, reações adversas e ajuste de dose de medicamentos utilizados por pacientes em hemodiálise. Saúde (Santa Maria), 2018; 44(3): 1-11.

25. SWARTZ MH. Tratado de semiologia médica. Elsevier Brasil, 2015; 794p.

26. VAIDOTAS $M$, et al. Erros de medicação em unidades de pronto atendimento: prontuário eletrônico, barreira eficaz? Einstein (São Paulo), 2019; 17(4): 1-5.

27. VINCENT JL. Annual update in intensive care and emergency medicine 2012. Springer Science \& Business Media, 2016, 876p. 